ARTICLE OPEN

\title{
Robust anomalous metallic states and vestiges of self-duality in two-dimensional granular In- $\mathrm{InO}_{x}$ composites
}

\author{
Xinyang Zhang $\mathbb{i D}^{1,2 凶}$, Bar Hen $\mathbb{D}^{3}$, Alexander Palevski $\mathbb{D}^{3}$ and Aharon Kapitulnik ${ }^{1,2,4}$
}

\begin{abstract}
Many experiments investigating magnetic-field tuned superconductor-insulator transition (H-SIT) often exhibit low-temperature resistance saturation, which is interpreted as an anomalous metallic phase emerging from a 'failed superconductor', thus challenging conventional theory. Here we study a random granular array of indium islands grown on a gateable layer of indiumoxide. By tuning the intergrain couplings, we reveal a wide range of magnetic fields where resistance saturation is observed, under conditions of careful electromagnetic filtering and within a wide range of linear response. Exposure to external broadband noise or microwave radiation is shown to strengthen the tendency of superconductivity, where at low field a global superconducting phase is restored. Increasing magnetic field unveils an 'avoided H-SIT' that exhibits granularity-induced logarithmic divergence of the resistance/conductance above/below that transition, pointing to possible vestiges of the original emergent duality observed in a true H-SIT. We conclude that anomalous metallic phase is intimately associated with inherent inhomogeneities, exhibiting robust behavior at attainable temperatures for strongly granular two-dimensional systems.
\end{abstract}

npj Quantum Materials (2021)6:30; https://doi.org/10.1038/s41535-021-00329-2

\section{INTRODUCTION}

An increasing number of recent experiments have been pointing to the possibility of zero temperature transition from a superconducting state to an 'anomalous metallic regime' with $T \rightarrow 0$ electronic properties that cannot be understood on the basis of conventional Fermi liquid/Drude theory (for a recent review see ref. $\left.{ }^{1}\right)$. In particular, it has been argued that the anomalous metal behaves as a 'failed superconductor,' a state in which there are significant superconducting correlations, yet the system fails to globally condense even as $T \rightarrow 0$, settling at a finite conductivity that can be orders of magnitude larger than the Drude conductivity. Among its striking features, current in the anomalous metallic regime is carried by bosonic quantum fluctuations of the superconducting order parameter, exhibiting giant positive magnetoresistance, a much suppressed Hall response ${ }^{2}$, and absence of cyclotron resonance ${ }^{3}$.

Anomalous metallic phases often emerge in searches for a zerotemperature superconductor-insulator transition (SIT) in disordered superconducting films, yielding instead a quantum superconductor-to-metal transition (QSMT), typically triggered by varying external parameters such as magnetic field, gate voltage, and degree of disorder ${ }^{2,4-12}$. Focusing on the magnetic-field tuned SIT (H-SIT), an 'avoided' transition (that is, a higher temperature signature of $\mathrm{H}$-SIT that gives way to resistance saturation at lower temperatures) is often found above the QSMT, accompanied by low-temperature resistance saturation that may persist on both sides of this transition. While much of the current focus is on anomalous metallic phases proximate to a QSMT, an anomalous metallic regime has also been ubiquitously identified on the insulating side of a putative SIT. Such behavior was identified for example in amorphous superconductors tuned by magnetic field ${ }^{6}$, or disorder ${ }^{13}$, and assumed to be originating from superconducting fluctuations that persist to the high resistance state. This scenario can be rationalized in the presence of inherent inhomogeneities, where a study of the effect of a ground plane next to the sample further suggested the importance of local superconducting phase coherence ${ }^{14}$.

From the very nature of the phenomenon, it is clear that the failed superconductor is extremely fragile, primarily due to the inhomogeneous nature of the superconducting state. This can be a result of a granular morphology, or a result of microscopic disorder that is 'amplified' in the superconducting state to yield an effective inhomogeneous microstructure ${ }^{15-17}$ or unstable nonequilibrium state due to fluctuations ${ }^{18}$. Indeed, recent experiments probed the stability of magnetic-field tuned superconducting amorphous indium-oxide ${ }^{19}$ and $\mathrm{MoGe}^{20}$ films, concluding that an observed metallic state can be largely eliminated by adequately filtering external radiation. Since in practice any such statement is subject to the limited sensitivity of experiment, the ultimate question arises whether in the absence of any external perturbation, the film's resistance will saturate to a finite resistance, may it be smaller than the experimental limit, as $T \rightarrow$ 0 . This challenging question arises following many experiments where attempts to eliminate the metallic phase failed, and by theoretical solution of a model of superconducting grains embedded in a metallic matrix, where such anomalous metallic behavior can occur in the neighborhood of a QSMT ${ }^{1}$. Thus, it is essential to distinguish a 'fragile superconductor,' which is sensitive to external perturbations, from an anomalous metal, i.e., a 'failed superconductor' in which quantum phase fluctuations preclude superconducting long-range-order even as $T \rightarrow 0$.

With the reasonable assumption that a superconducting transition in 2D disordered metallic films is dominated by phase

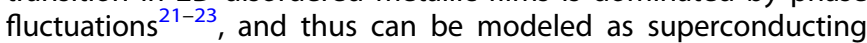
grains embedded in a metallic matrix, we may search for a system where phase fluctuations are enhanced, and material parameters can be tuned to allow interrogation of an observed anomalous metallic phase within the sensitivity of the experimental system.

\footnotetext{
${ }^{1}$ Geballe Laboratory for Advanced Materials, Stanford University, Stanford, CA, USA. ${ }^{2}$ Department of Applied Physics, Stanford University, Stanford, CA, USA. ${ }^{3}$ School of Physics and Astronomy, Raymond and Beverly Sackler, Faculty of Exact Sciences, Tel Aviv University, Tel Aviv, Israel. ${ }^{4}$ Department of Physics, Stanford University, Stanford, CA, USA.

凶email: xinyangz@stanford.edu
} 
a

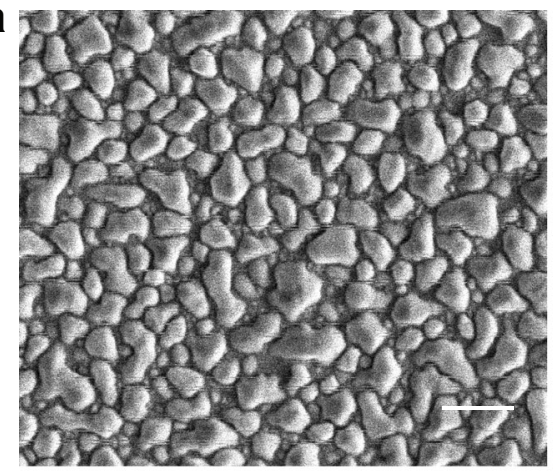

C

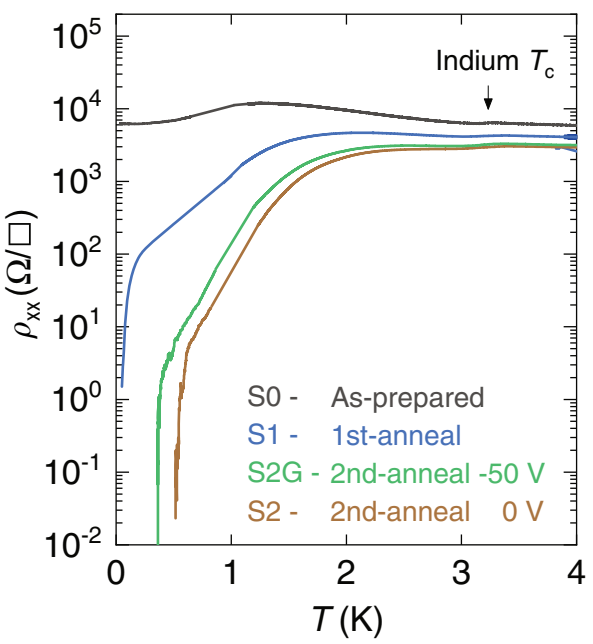

b

Equivalent diameter $(\mu \mathrm{m})$

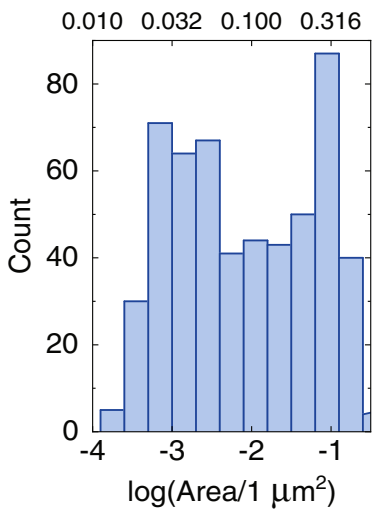

d

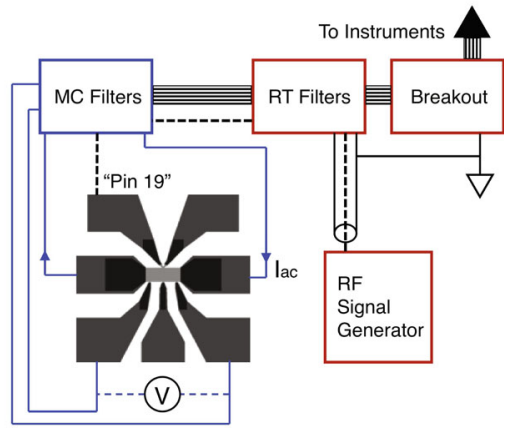

Fig. 1 Sample characterization. a Scanning electron microscopy (SEM) micrograph of the $\ln \mathrm{O}_{x} / \ln$ sample. Bright grains on the foreground are metallic indium, while dark background that lies uniformly underneath is weakly-insulating amorphous InO $\mathrm{O}_{x}$. Note the existence of In grains of all scales including the interstitial ones. Scale bar at lower right corner indicates $1 \mu \mathrm{m}$. b Histogram of logarithm of grain area, extracted from the SEM data using a clustering algorithm (see Supplementary Fig. 1), shows a broad distribution of grain size. The top axis shows equivalent grain diameter. Limitation of image resolution cuts off the distribution below $\sim 0.01 \mu \mathrm{m}$. c Zero-field resistivity as a function of temperature (smoothed for visibility). Superconducting transition temperature $\left(T_{c} \approx 3.4 \mathrm{~K}\right)$ of indium is indicated by the arrow where resistivity drops by around $10 \%$ for all anneal stages. As sample anneals, resistivity decreases and a global superconducting ground state emerges. d Schematics showing resistance measurement and coupling of RF signal to the sample. See "Methods" for filter specifications. The RF signal generator generates at output power $0 \mathrm{dBm}(1 \mathrm{~mW})$ unless otherwise noted. 'Breakout' is a room-temperature breakout box for measurement wires, where the shielding serves as a common ground for the entire measurement system.

Since quantum fluctuations of the phase of an isolated superconducting grain are associated with the charging energy, which is further controlled by the dielectric response of the surrounding matrix, a properly designed granular system should be our starting point.

In this paper, we examine the robustness of an observed metallic phase in $2 \mathrm{D}$ InO $\mathrm{O}_{x} / \mathrm{In}$ composite system, where a thin layer of amorphous indium-oxide $\left(\ln \mathrm{O}_{x}\right)$, tuned to be 'barely metallic' and non-superconducting, provides the Josephson-coupling between indium (In) islands grown on top of it. The unique microstructure of the composite allows for fine control of the fragility of the zero-field superconducting state. Furthermore, the relatively large In grains ensure thermal equilibrium, and thus global superconductivity is achieved uniquely via phase coherence. This system thus yields a highly tunable yet robust anomalous metallic state. While this metallic phase is observed under careful electromagnetic filtering and within a wide range of linear response, exposing it to external broadband noise or microwave radiation is shown to strengthen the tendency of superconductivity. Furthermore, in a wide range of parameters, the external radiation restores a true superconducting phase (within the sensitivity of the measurements) which is a direct consequence of enhanced phase coherence.

Close to the QSMT in the metallic phase, resistance saturates to a value that is much smaller than the normal state 'Drude value,' and depends on magnetic field as a power law. Increasing the magnetic field, a logarithmic divergence of the conductance is observed, with isotherms merge into an 'avoided' H-SIT (that is, a higher temperature signature of $\mathrm{H}$-SIT that gives way to resistance saturation as the temperature is lowered). Further increase of the magnetic field yields an anomalous logarithmic divergence of the resistance with a large coefficient ${ }^{24}$, which mimics the conductance behavior at lower magnetic fields, and thus suggests some form of duality between the low-field and high-field sides of the crossing point. The origin of the logarithmic behavior can be attributed to the granular nature of the system ${ }^{25}$.

Finally, we note that this versatile system was recently shown to undergo a true magnetic field tuned SIT $(\mathrm{H}-\mathrm{SIT})^{26}$, similar to that of uniform $\operatorname{InO}_{x}$, exhibiting a 'giant' magnetoresistance above the $\mathrm{H}$ $\mathrm{SIT}^{27,28}$ and critical behavior that manifests the duality between Cooper pairs and vortices ${ }^{29}$. This in turn will be a key starting point to our discussion below on 'avoided' H-SIT. 

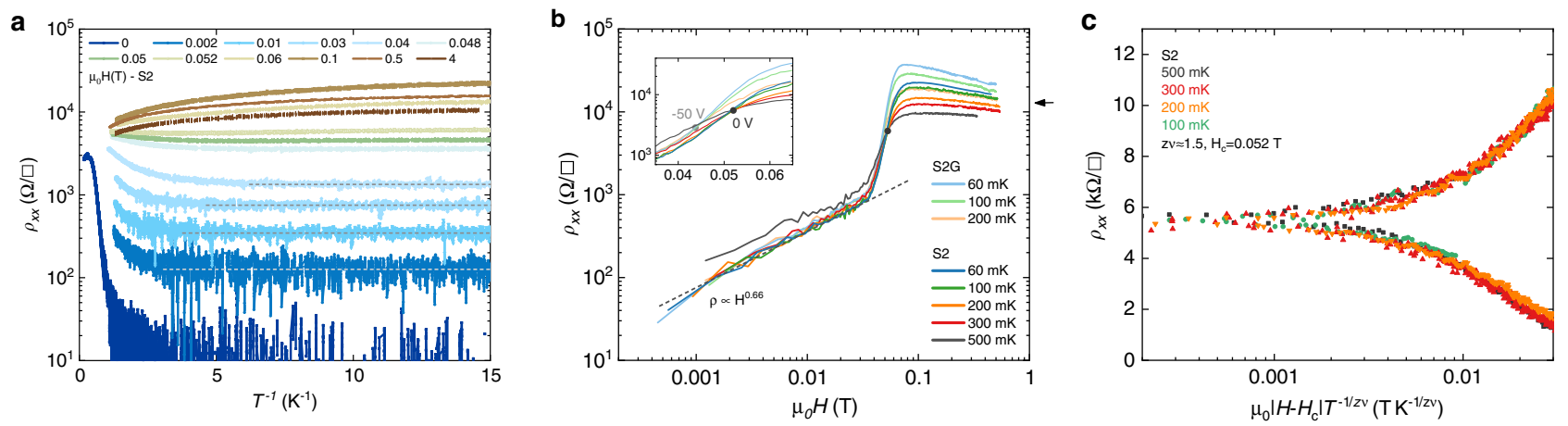

Fig. 2 Anomalous metallic phase and the avoided H-SIT. a Arrhenius plot of resistivity in various magnetic fields for sample S2. Robust resistivity saturation can be found in low magnetic field at as high as $300 \mathrm{mK}$, indicated by dashed horizontal lines. b Log-log plot of magnetoresistance (MR) at different temperatures for S2 and S2G. In anomalous metallic phase between 0 and 0.04 T, resistivity is essentially temperature-independent below $300 \mathrm{mK}$. The data collapse into a single power law $\rho \propto H^{0.66}$, indicated by the dashed line. The arrow indicates resistivity of S2 at $4 \mathrm{~T}$ and $60 \mathrm{mK}$. (Inset) Expanded view of the same data between 0.035 and $0.065 \mathrm{~T}$ shows isotherm crossing points (solid circles) separating metallic from insulating behavior. c Scaling function for resistivity $\rho\left(\left|H-H_{c}\right| T^{-1 / z v}\right)$ of S2 at 100, 200, 300, and 500 mK. The best data collapse occurs for $z v \approx 1.5$.

\section{RESULTS AND DISCUSSION General trends}

As-grown $\ln \mathrm{O}_{x} / \mathrm{In}$ samples were prepared intentionally to be initially non-superconducting. Subsequent room-temperature annealing in vacuum with minimal air contact reduced resistivity as demonstrated for 4 sample anneal stages in Fig. 1c: SO-as prepared sample, S1-the sample after 1st anneal, S2-the sample after a subsequent anneal, and S2G-the second-anneal sample with a $-50 \mathrm{~V}$ back gate applied. For every anneal stage including the back-gated, sample resistivity increases when cooled from 300 to $3.2 \mathrm{~K}$ similar to plain $\mathrm{InO}_{x}$. Around $3.2 \mathrm{~K}$, however, resistivity drops by $\sim 10 \%$ marking the onset of superconductivity in In grains. Upon further cooling, SO resistivity fails to exhibit global superconductivity, but instead saturates as $T \rightarrow 0$. (Such samples, which do not show a zero-field global superconductivity, will be discussed in a different publication.) As the sample anneals, zero-resistance is almost achieved at base temperature for $\mathrm{S} 1$, and can be clearly identified at 0.5 and $0.35 \mathrm{~K}$ for S2 and S2G, respectively. Hall measurements on the annealed samples confirmed a high carrier density of $4 \times 10^{15} \mathrm{~cm}^{-2}$. This value, which is higher but close to that of the pure $\operatorname{lnO}_{x}$, is what we expect for a composite system where the non-percolating component exhibits much lower resistance and much higher carrier density. (See Supplementary Note 1 for an estimation of effective carrier density.) Thus, while the $-50 \mathrm{~V}$ back gating effectively reduced carrier concentration leading to a reduction in $T_{c}$, it was not enough to tune S2 through the QSMT. We henceforth focus on S2 and S2G to study the transition between a superconducting and its proximate ground states in a perpendicular magnetic field.

With the application of perpendicular magnetic field, the zeroresistance superconducting state in the $\mathrm{In} / \mathrm{InO}_{x}$ system is rapidly terminated at a magnetic-field-tuned QSMT, and an anomalous metallic phase is identified for $0 \lesssim \mu_{0} H<0.04 \mathrm{~T}$. Figure $2 \mathrm{a}$ shows resistivity that saturates as $T \rightarrow 0$ at a level orders of magnitude lower than the Drude value. As magnetic field increases, resistivity initially rises as a power law that is essentially temperatureindependent below $300 \mathrm{mK}$ for both S2 and S2G (see Fig. 2b). Then, at $H \approx 0.04 \mathrm{~T}$, resistivity quickly picks up, leading to a giant magnetoresistance (MR) peak. The transition between saturating and diverging temperature dependence of resistivity is marked in the inset of Fig. $2 \mathrm{~b}$ at 0.052 and $0.043 \mathrm{~T}$, for S2 and S2G, respectively. This is reminiscent of a true $\mathrm{H}$-SIT, with a transition to boson-dominated insulating ground $\operatorname{state}^{27,28}$. At $H \approx 0.08 \mathrm{~T}$, resistivity peaks at $40 \mathrm{k} \Omega / \square$ at the highest. Beyond the peak, resistivity slowly decreases up to $8 \mathrm{~T}$, but remains orders of magnitude larger than the normal state value, indicating the persistence of superconducting correlation well into the insulating state $^{28,30}$. Qualitatively, SO and S1 behave similarly as shown in Supplementary Fig. 2, but have much higher resistivity up to $0.5 \mathrm{M} \Omega / \square$ at the peak.

\section{Low-field regime: a failed superconductor}

Starting with the low-field regime, where a failed superconducting phase yields a saturated resistance, the temperature-independent power-law MR before the rapid upturn, $\rho(H) \propto H^{0.66}$, fully overlaps with the anomalous metallic regime. Exponentiating this behavior, this is equivalent to a quantum-tunneling expression with $\rho(H) \propto \exp \left(-U(H) / k_{\mathrm{B}} T_{\text {eff }}\right)$, previously observed in amorphous MoGe films ${ }^{5}$, where the logarithmic field dependence of $U(H)$ was attributed to activation of free dislocations in a vortex lattice with short spatial correlations ${ }^{31}$. Since this mechanism should strongly depend on the morphology of the film, it is no surprise that the magnetoresistance curves in the anomalous metallic phase for both S2 and S2G films collapse on the same curve in Fig. 2b. Slight differences between the two samples are observed as the resistance increases above saturation toward a putative crossing point, which we will dub as 'avoided true H-SIT.' We will come back to analyze the form of the resistance increase.

\section{Avoided H-SIT}

Beyond the resistivity upturn at $H \approx 0.04 \mathrm{~T}$, isotherm crossing points that separate positive MR on the low-field regime from positive MR on the high-field regime are observed (marked by full circles) in the inset of Fig. 2b. This type of behavior would describe a true H-SIT if indeed a true superconducting phase would be attained, accompanied by divergence of the resistance on the insulating side. Since resistance saturation is observed in the lowtemperature limit of both regimes, we may consider this avoided criticality as a crossover and attempt scaling at higher temperatures (here $T>100 \mathrm{mK}$ ), before the system is dominated by the anomalous metallic phase. Similar approach was demonstrated previously $^{6,32}$ to lead to scaling exponents of order $z v \approx 1.5$ when we scale the data according to $\rho(T, H)=\rho_{c} \mathcal{F}\left(\left|H-H_{c}\right| T^{-1 / z v}\right)$ in Fig. 2c. It is also interesting to note that such a behavior with a similar critical exponent was previously observed in a 2D system of aluminum islands coupled by $2 \mathrm{DEG}^{11}$, thus can be added to the list of common features between systems of induced superconducting granularity in otherwise presumed homogeneous materials such as amorphous MoGe $\mathrm{e}^{5,6}$ and systems with extreme granularity in ordered ${ }^{11}$ and disordered (this manuscript) films. 

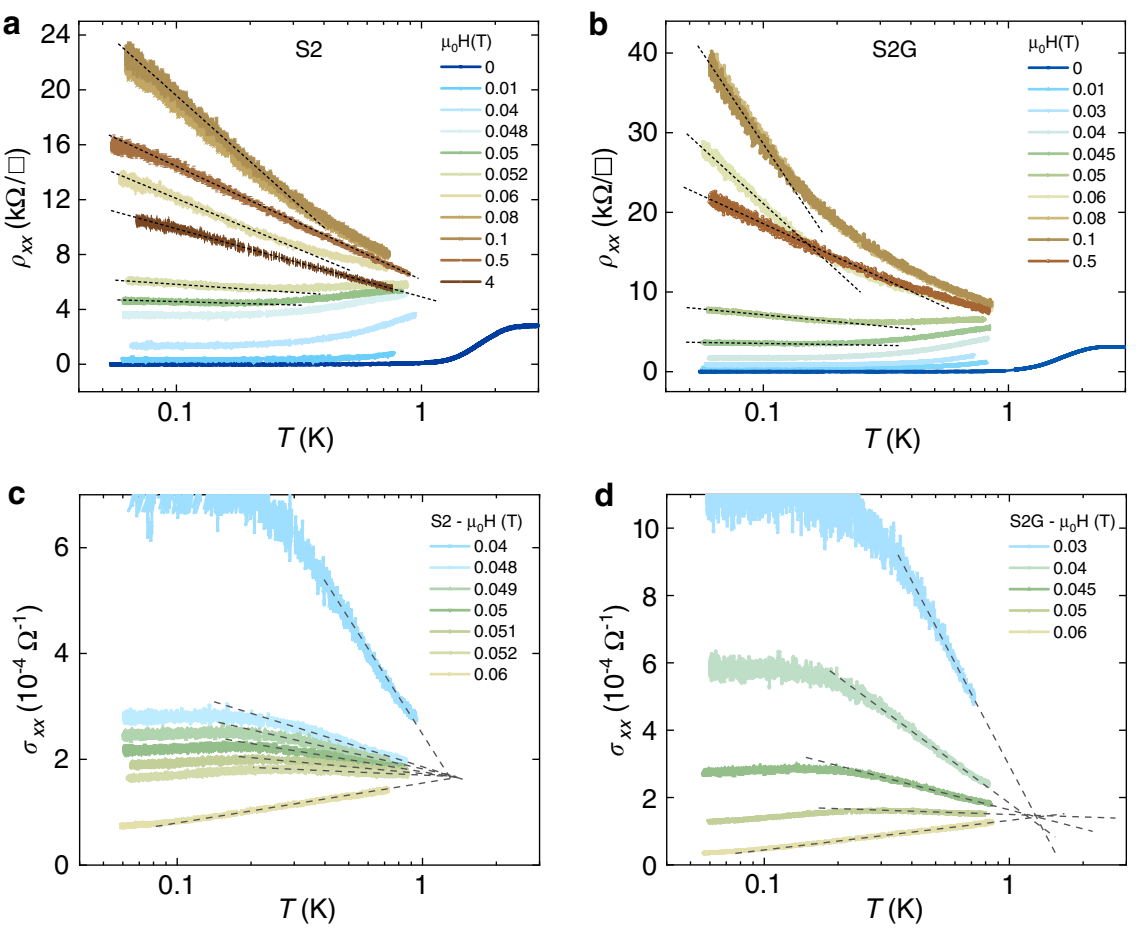

Fig. 3 Logarithmic divergence of resistivity and conductivity proximate to the avoided H-SIT. a, b Resistivity versus logarithm of temperature in perpendicular magnetic field, for anneal stage S2 and S2G, respectively. Note the difference in resistivity scales. Dotted lines are guidance to the eye, demonstrating the logarithmic divergence of resistivity over a broad temperature range. c, $\mathbf{d}$ Conductivity versus logarithm of temperature for S2 and S2G, obtained by inverting the data in (a) and (b). Logarithm divergence of conductivity is found before the saturation sets in at low temperature.

\section{Behavior proximate to the 'avoided H-SIT'}

Examination of the resistance trends on both sides of the crossing point reveals an intriguing behavior which may indicate the existence of vestiges of duality despite it being an 'avoided transition.'

Starting with the 'insulating regime', again S2 and S2G show similar behavior, exhibiting anomalous logarithmic divergence of resistivity that is much weaker than the commonly observed activation or variable-range hopping trends. As shown in Fig. 3a, b, where resistivity is plotted versus logarithm of temperature, within a broad range of temperature, the data can be fit by a straight line in the form $\Delta R / R(1 \mathrm{~K})=\mathrm{A} \cdot \mathrm{R}(1 \mathrm{~K}) \ln (1 / \mathrm{T})$, where the slope $A=$ $(8.44 \pm 0.12) \times 10^{-5} \Omega^{-1}$ for $S 2$ at $4 \mathrm{~T}$. Slopes with similar magnitude are found for other magnetic fields, and for the different anneal stages. The data and particularly the slopes of the log- $T$ behavior are neither compatible with Kondo-effect behavior, nor are they compatible with weak localization corrections. Similar logarithmic divergence has been observed in the high-field insulating state of underdoped $\mathrm{La}_{2-x} \mathrm{Sr}_{x} \mathrm{CuO}_{4}{ }^{33}$, and in amorphous $\operatorname{lnO}_{x}{ }^{24}$. Invoking inhomogeneous microstructure for these otherwise considered homogeneous materials, a possible connection between granularity and logarithmic divergence of the temperature-dependent resistivity has been previously discussed (see e.g., ref. ${ }^{25}$ ). We further note that for magnetic fields close to the crossing point, the logarithmic divergence tends to saturation, while increasing the magnetic field beyond the magnetoresistance peak seems to recover a 'normal' insulating behavior commensurate with the initial $2 \mathrm{D}$ resistivity of the film before superconductivity sets in. Since the high-field resistivity is much smaller than the peak in magnetoresistance, we interpret the saturation on the insulating side as an increase in local phase coherence due to remnant superconductivity.

Turning to the transition to the 'anomalous metallic regime' below the putative crossing point, we find an anomalous logarithmic divergence of the conductivity (calculated as the inverse resistivity since the Hall contribution is negligible), that is much weaker than activation or variable-range hopping commonly found where a true H-SIT is observed ${ }^{2,26}$. As shown in Fig. $3 c$, d, where conductivity is plotted versus logarithm of temperature, within a broad range of temperature, the data can be fit by a straight line. Similar to the insulating regime, this logarithmic divergence exhibits large prefactor, which again deems it as a crossover to the exponential divergence rather than a weak correction to a normal state conductivity.

Figure 3 is a striking demonstration of vestiges of the H-SIT quantum critical point in this 'avoided transition'. It further makes the connection between the resistance saturation on the superconducting side and that on the insulating side, where in our highly granular films it can be attributed to vestiges of local phase coherence in strongly fluctuating superconducting grains.

Finally, we turn to making a more microscopic connection between the logarithmic divergence and the morphology of our films. We will then argue that this connection can be generalized to samples where strong inhomogeneity emerges in the superconducting state following amplification of local disorder ${ }^{15-17}$. Focusing on the insulating side, here, with a carefully analyzed microstructure, we are able to show that the observed logarithmic divergence is indeed consistent with originating from the extreme granular inhomogeneity, leading to weak resistivity divergence over an intermediate range of temperature. As shown in Fig. 1b, the granular structure consists of a hierarchy of grain sizes, spanning over two orders of magnitude, from microscopic interstitial ones to 'large grains'-a fraction of a $\mu \mathrm{m}$. Hence, in the insulating state, the system consists of a wide range of tunneling barriers between the metallic (or superconducting) islands. These barriers are determined by a competition between normal or Josephson tunneling and charging energy mediated by the dielectric background environment. Assuming a log-uniform distribution of barrier's energy $\Delta: p(\Delta)=1 / \Delta$ and integrating over 


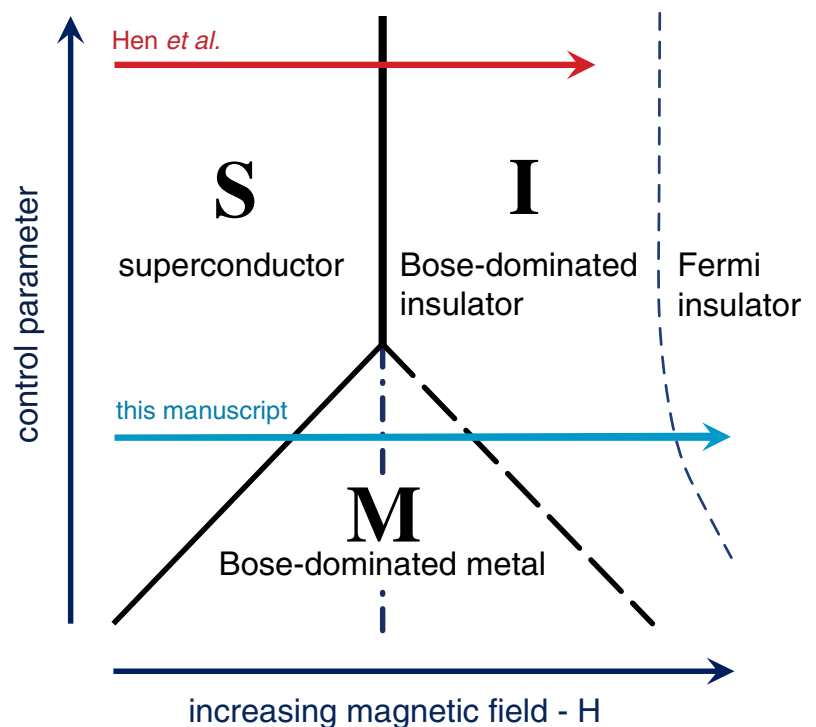

Fig. 4 Phase diagram of superconductor-metal-insulator in 2D. Phase diagram is drawn as a compilation of the data presented in Hen et al. ${ }^{26}$ where true H-SIT is observed, together with the results discussed in this manuscript. The thick solid line represents a true $\mathrm{H}$ SIT, with its associated critical behavior and emerging self-duality ${ }^{29}$. At low field and small control parameter, an intervening metallic phase emerges from a quantum superconductor-to-metal transition (solid line), showing Bose character until it fades at a crossover to a stronger, Bose-dominated insulating phase (dashed line). At higher magnetic field pairing is quenched and Fermi-dominated insulating behavior is recovered (thin dashed line). Dash-dotted line represents a line that separates the two regimes of metallic phase, where vestiges of self-duality are observed (see text).

parallel conductances, we show that the resultant resistance can exhibit weak power law or logarithmic divergence. At much lower temperature, however, the resistivity will diverge much faster in an activated fashion. A more detailed account of our analysis is shown in Supplementary Fig. 3. This emerging behavior further supports our intuition that transport is determined by a collective effect of the In grains coupled within the $\operatorname{lnO}_{x}$ matrix.

\section{Phase diagram}

It was recently demonstrated that where true H-SIT is observed, the quantum phase transition exhibits the emergence of selfduality with an exponential diverging variable-range hoping behavior of the resistivity above, and the conductivity below, the transition ${ }^{26,29}$. The insulating phase was then identified as a Hall insulator, while the superconducting phase would be an equivalent phase for vortices. Moreover, the assembly of the critical behavior effects was found to be similar to that of quantum Hall to insulator transition ${ }^{34}$, particularly the critical exponents and the emergence of self-duality ${ }^{26,29}$. In fact, in attempting to summarize the above results into a phase diagram we observe further similarity between the two phenomena.

Figure 4 is a phase diagram for the $\ln / \ln \mathrm{O}_{x}$ system based on the experiments of Hen et al. ${ }^{26}$ observing a true $\mathrm{H}$-SIT and the present data showing an intervening metallic phase. The vertical axis represents an external parameter that controls the nature of the critical behavior. While in uniform films it could be the disorder, in the presence of strong granularity it may represent the distribution of the intergrain couplings. Where material parameters are tuned to observe an anomalous metal (e.g., weak intergrain couplings), a putative crossing point is observed as well with a non-universal critical resistance and different critical exponents, typically $z v \approx 1.5$. The fact that the resistance above the crossing
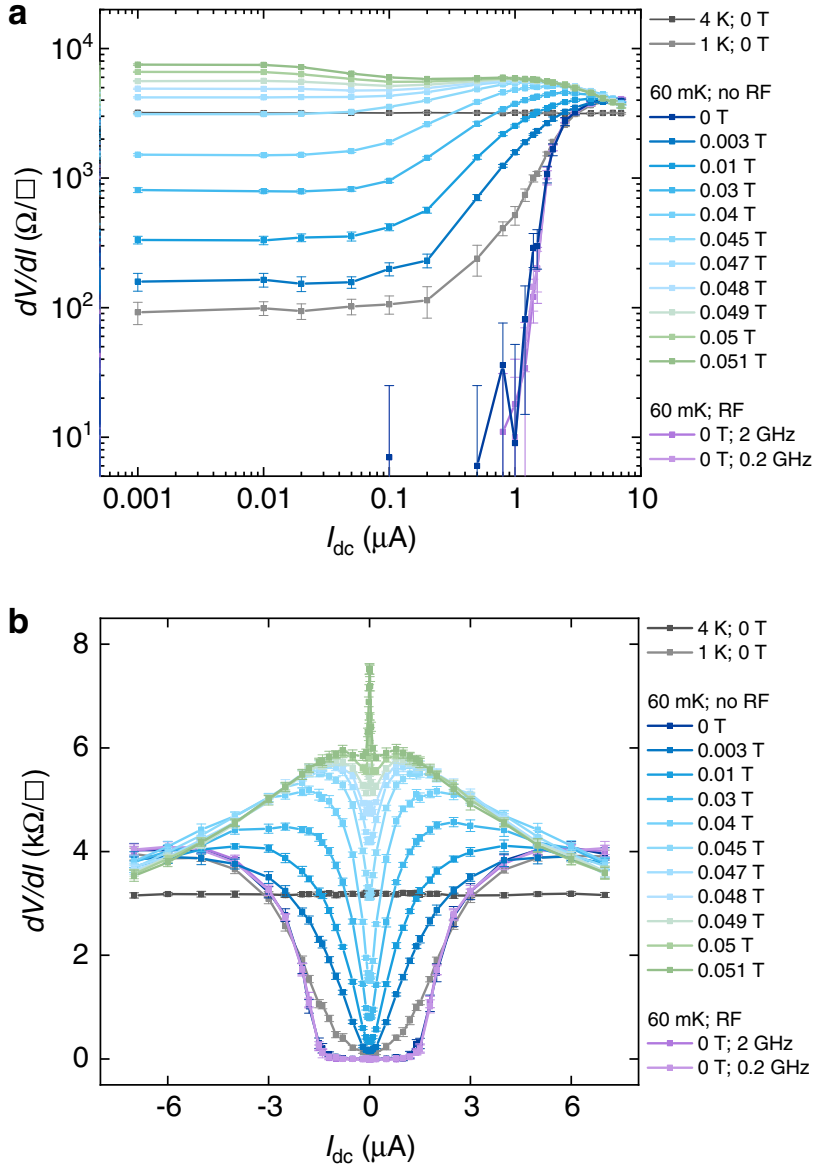

Fig. 5 Differential resistivity in perpendicular magnetic field or subject to RF injection. a Differential resistivity $d V / d l$ versus direct current bias for S2G at $60 \mathrm{mK}$, showing a superconducting critical current of about $1 \mu \mathrm{A}$. Injection of RF signal of $2 \mathrm{GHz}$ (dark purple) and $0.2 \mathrm{GHz}$ (light purple) leads to a slight increase in critical current. Linear response below at least $20 \mathrm{nA}$ is demonstrated in the anomalous metallic phase. Dark gray curve is $d V / d l$ at $4 \mathrm{~K}$, marking the normal state value. b Same data shown in full linear scale. Zerobias features evolve from a dip at low field to a sharp peak at higher field, corresponding to the anomalous metallic phase and insulating phase, respectively. Error bars indicate standard deviation.

resembles the conductance below the crossing, where both lead to a regime of saturated resistance suggests that vestiges of the self-duality line that emerges at the true H-SIT remain also in the regime where a metallic phase emerges, hence marks two dual regimes of anomalous metallicity. This observation is marked with the dash-dotted line in the phase diagram. Thus, while for true $\mathrm{H}$ SIT a $\sigma_{x x} \sim \exp \left[\left(\Delta_{S} / T\right)^{\delta}\right]$ on the superconducting side transforms to $\rho_{x x} \sim \exp \left[\left(\Delta_{l} / T\right)^{\delta}\right]$ in the insulating side ${ }^{29}$ (here $\Delta_{s}$ and $\Delta_{l}$ are activation gap scales for the superconducting and insulating regimes, respectively). At weaker control parameter a $\sigma_{x x} \sim$ $\ln \left(\Delta_{S} / T\right)$ that appears following the resistance saturation crosses over to $\rho_{x x} \sim \ln \left(\Delta_{l} / T\right)$ with increasing magnetic field. The insulating side then exhibits further increase of the resistance before pairing is quenched and Fermi-dominated insulating behavior is recovered.

\section{Response to external radiation}

Despite extensive filtering at different stages of our dilution refrigerator, the anomalous metallic phase appears robust. Nonetheless, fragility of the underlying superconducting state can cause this inhomogeneous system to be extremely sensitive to 
environmental perturbation. In a given magnetic field, barriers associated with collective vortex effects are established throughout the sample, leading to a saturated resistance commensurate with that field. However, it is important to explore whether the observed resistance is due to nonequilibrium effects where, for example, electrons fail to thermalize with the lattice due to poor electron-phonon coupling, or external noise heats the electrons, thus obstructing coherence in weaker Josephson couplings and disrupting global superconductivity. We already discussed the unique microstructure of our samples, where the large In grains ensure good thermalization of the local pair amplitudes. Indeed, Fig. 5a shows linear response of the resistance measurements in the anomalous metallic regime over a wide range of current bias. Non-linearities are only observed at higher currents, and are presumably associated with junctions locally exceeding their critical current.

Having established linear response, we further need to explore the possible influence of external radiation on the occurrence of anomalous metallicity. To test for this effect, we may introduce external noise by removing successive layers of filtering in a controlled fashion, or by injecting radio-frequency (RF) signal into the sample and measure its response in resistivity.

We emphasize that our objective is not to study straightforward effects of well-coupled radiation to the sample (see discussion below), but rather to inject radiation into various ports that lead to the sample or sample space in order to mimic poor decoupling of the low-temperature measurement from the external world. This is important in view of recent studies that suggest that some observations of anomalous metallic state are a consequence of poor filtering and enhanced coupling to the external world.

In Fig. 6, we show Arrhenius plot of resistivity under different experimental conditions. By either introducing $2 \mathrm{GHz}$ signal or bypassing the room-temperature low-pass filter (see Fig. 1d), resistivity is lowered by as much as $\sim 25 \%$ at $0.03 \mathrm{~T}$. This is contrary to the electron heating scenario, where an elevated electron temperature would raise saturation resistance. In an extreme case, at a field strength of $0.0002 \mathrm{~T}$ or 2 Gauss, the anomalous metallic behavior is suppressed completely and true superconductivity is recovered, where resistivity drops below our measurement sensitivity (see the lower panel in Fig. 6). It is interesting to note that in a completely different system of nanopore-modulated YBCO thin films, where anomalous metallic state has been observed, the removal of filtering resulted in a similar reduction of the saturated resistance at low temperatures ${ }^{35}$. This result was taken as a proof of true cooling of electrons in that highly inhomogeneous system. However, the introduction of deliberate microwave radiation in addition to removal of parts of the filtering system can further illuminate the nature of the robustness of the metallic phase and in particular the effect of re-entrant superconductivity.

Discovered over 50 years ago, microwave-enhanced superconductivity has been studied experimentally in constrainttype ${ }^{36,37}$ and superconductor-normal-superconductor (SNS) Josephson junctions ${ }^{38,39}$. Dubbed as 'microwave-stimulated superconductivity' ${ }^{40}$, this phenomenon was found to be ubiquitous in studies of SNS weak links. Initial theoretical understanding of this phenomenon invoked a nonequilibrium gap-enhancement proposed by Eliashberg ${ }^{41}$. However, more recent analyses, especially taking into account proximity-effect under external radiation, concluded that much of the enhancement of critical current in such SNS junctions arises from enhanced phase coherence (for a recent review see Klapwijk and de Visser ${ }^{40}$ ).

As demonstrated above, global superconductivity in the $\ln / \ln \mathrm{O}_{x}$ system is established via phase coherence among the In grains, coupled through the underlying $\operatorname{lnO}_{x}$ layer. In the presence of magnetic field, pinned vs. mobile collective vortex effects reflect the restoration or loss of local phase coherence, respectively. Thus, a metallic state appears over a zero-resistance superconducting state when a vortex-induced global phase slip appears-marking
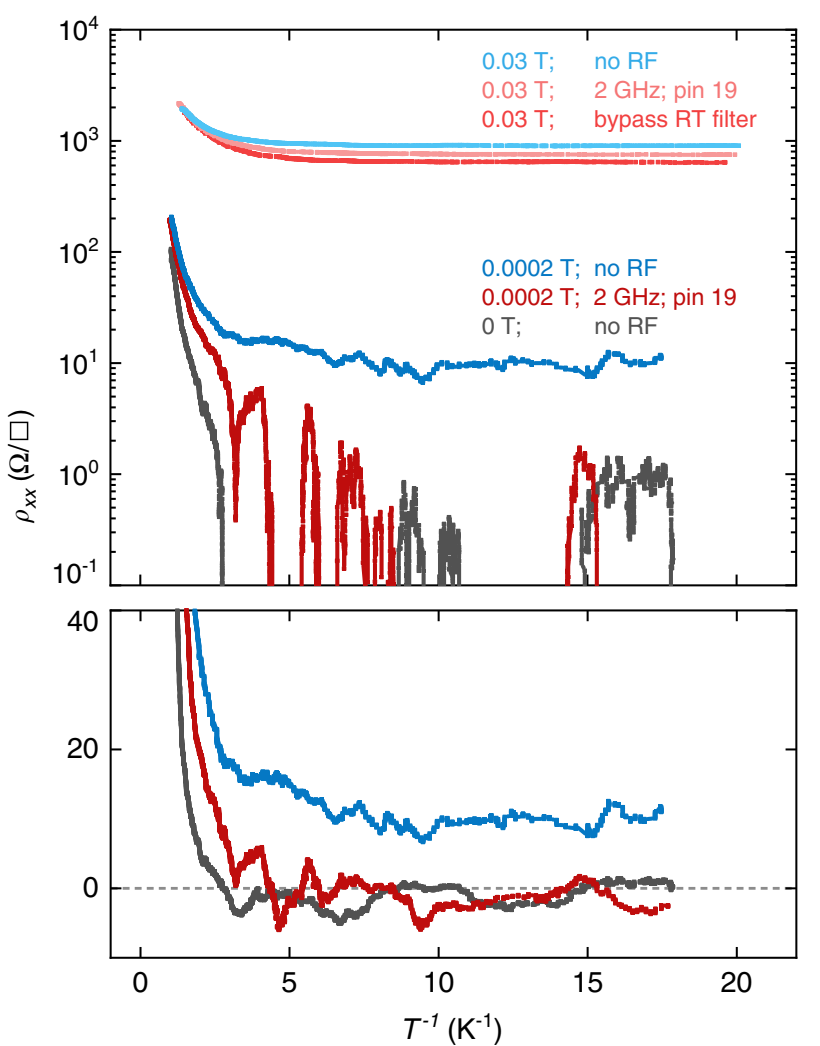

Fig. 6 Radio-frequency (RF) signal and broadband noise enhancing tendency toward superconductivity. Enhanced conductivity by coupling RF signal to the sample S2G, at 0.03 and $0.0002 \mathrm{~T}$. In the latter case, a zero-resistance state emerges as a result of injecting $2 \mathrm{GHz}$ signal. Lower panel shows $0.0002 \mathrm{~T}$ data in linear scale, further demonstrating the effect of RF injection. 'No RF' means zero output power at RF signal generator. 'Pin 19' denotes a voltage lead connected to the sample in the middle along the Hall bar, see Fig. 1d. 'Bypass RT filter' means RT filter is bypassed and broadband noise enters signal line. (No RF signal is injected in this case; data smoothed for higher visibility.) Other wires are checked for decoupling from the sample at RF. See Supplementary Figs. 4 and 5 for an estimate of RF power transmitted to the sample and RF injection results in insulating state.

the loss of global phase coherence. However, the emerging metallic phase retains much of the character of the superconducting phase, where significant superconducting correlations are present, thus establishing a resistance much lower than the respective 'Drude resistance'. The microwave-enhanced conductivity of this metallic state, and the restoration of the superconducting state at very low fields are a manifestation of the robustness of this failed superconducting state.

We return to Fig. 5, which shows differential resistivity $d V / d I$ in presence of a direct current bias. In zero magnetic field, superconductivity is quenched by a critical current of $1 \mu \mathrm{A}$, which is slightly enhanced by application of RF signal. In an external magnetic field, $d V / d l$ yields a zero-bias minimum value in low field, and a zero-bias peak in higher field, more clearly shown in full linear scale in Fig. 5b.

The evolution of the differential resistivity behavior displayed in Fig. 5 has been observed previously in a 2D system of aluminum islands, coupled with a gated 2D electron gas ${ }^{11}$. Such a system may be considered an ordered array of islands, as shown by the oscillations in the array's resistance at integer and certain fraction of flux quanta entering their periodic sample. While the overall behavior of the two systems is the same, the random distribution of the In islands in our system blurs any possible ordered 


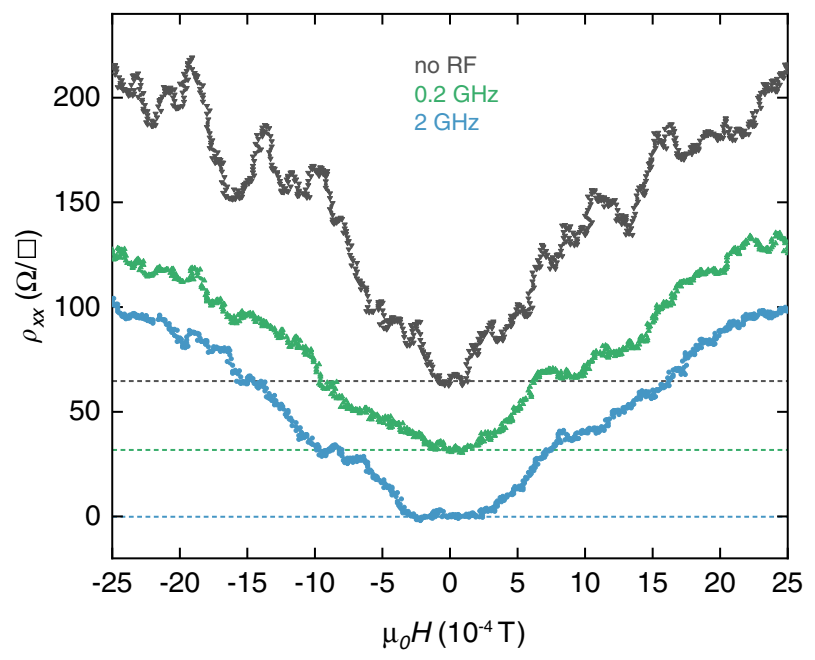

Fig. 7 Magnetoresistance of S2G subject to RF signal injection at various frequencies. Resistivity as a function of magnetic field for $\mathrm{S} 2 \mathrm{G}$ at $60 \mathrm{mK}$. RF signal of various frequencies is injected into the sample. From top to down: no RF, $0.2 \mathrm{GHz}$, and $2 \mathrm{GHz}$. $\rho=0$ baseline (dashed line) of each curve is shifted for clarity.

oscillation in the magnetoresistance, although it may explain the non-smooth MR data to be discussed in the next section.

\section{Flux effects}

Figure 7 shows the effect of RF radiation on magnetoresistance. When no RF signal is present, as a result of the sample's extreme sensitivity to external magnetic field, we cannot clearly identify a lower critical field of a QSMT. Beyond the limitations in measurement sensitivity and magnet field resolution, the main observation is the large fluctuations in MR, up to $30 \%$ of the averaged behavior. Such repeatable fluctuations are likely a consequence of flux commensuration effect allowing flux quanta into the granular structure. While such an effect shows precise periodicity in ordered array of coupled grains, such as in the coupled array of $\mathrm{Al}$ islands ${ }^{11}$, or in studies of square aluminum wire networks in a magnetic field ${ }^{42}$, here the area enclosed by loops connecting indium islands through intergrain coupling is random, and the smeared oscillations we observe are a consequence of the mesoscopic nature of the effect, which in turn is also responsible for the observed low critical field. For example, the deep minimum at $\sim 15 \mathrm{G}$ corresponds to a flux quantum fitting in a loop of average diameter $\sim 1.3 \mu \mathrm{m}$, which in inspecting Fig. 1a, b, would correspond to a fundamental loop that includes the larger grains. Indeed, similar effects have been observed in granular cuprates ${ }^{43}$, inhomogeneous nanowires ${ }^{44}$, and granular lead-film bridges ${ }^{45}$.

The fact that the MR fluctuations are related to flux penetration through loops of grains connected by junctions is further evidenced from the effect of radiation. As we demonstrated above, the intergrain coupling in the $\ln / \ln \mathrm{O}_{x} / \mathrm{ln}$ junctions in our films is enhanced in the presence of microwave radiation, which in turn should make the films effectively more homogeneous and thus reduce the MR fluctuations. Indeed, Fig. 7 shows the suppression of the MR fluctuations, accompanied by enhancement in critical field in the presence of 0.2 or $2 \mathrm{GHz}$ RF radiation.

In summary, we examined the robustness of an observed metallic phase in $2 \mathrm{D} \operatorname{lnO}_{x} /$ In composite system. We found that we can tune this system to a regime of fragile superconductivity that upon application of a weak magnetic field becomes an anomalous metal. While our results are obtained under conditions of careful electromagnetic filtering and within a wide range of linear response, exposure to external broadband noise or microwave radiation is shown to strengthen the tendency of superconductivity, where at low field a global superconducting phase is restored. Increasing the magnetic field exposes an 'avoided H-SIT', which above that transition is characterized by an anomalous logarithmic divergence of the resistance, while below the transition a logarithmic divergence of the conductivity is observed. This further highlights the granular nature of the system, and points to possible vestiges of the original duality observed in a true H-SIT. We are then led to conclude that anomalous metallic phase is intimately associated with inherent inhomogeneities, exhibiting robust behavior at attainable temperatures for strongly granular two-dimensional systems.

\section{METHODS}

\section{Sample preparation}

Extreme granular inhomogeneity that features a broad distribution of grain size is achieved by depositing poorly wetting In metal onto uniform amorphous $\mathrm{InO}_{x}$ thin film. The underlying layer of $300-\AA \AA \mathrm{InO}_{x}$ was electronbeam evaporated onto a commercial lithium-ion conductive glass-ceramics ( $\mathrm{Li}^{+}-\mathrm{ICGC}$ ) substrate (MTI corporation) that enables depletion of electrons up to a $2 \mathrm{D}$ carrier density of $\sim 10^{14} \mathrm{~cm}^{-2}$ in back-gating configuration, as was also found in a recent study ${ }^{46}$. A deposition rate of $0.32 \AA / s$ in an oxygen partial pressure of $9.5 \times 10^{-6}$ Torr yielded a weakly insulating film. Without interrupting the vacuum, at a base pressure of $1 \times 10^{-7} \mathrm{Torr}$, indium was evaporated in situ at a rate of $5 \AA / \mathrm{s}$ for $100 \mathrm{~s}$, yielding a nominal $500-\AA$ layer of granular indium. The granular structure was confirmed by scanning electron microscopy as shown in Fig. 1a along with a histogram showing grain size distribution in Fig. 1b. The composite film was then patterned in Hall bar geometry $(200 \mu \mathrm{m} \times 100 \mu \mathrm{m})$ using photolithography, before titanium/gold contacts were subsequently patterned onto the sample. Great care was taken to keep the sample below $50^{\circ} \mathrm{C}$ at all times to preserve the amorphous nature of the underlying $\operatorname{InO}_{x}$. Effective tuning of disorder, manifested by slow decrease in resistivity, is achieved by annealing the sample in vacuum at room temperature.

\section{Resistivity measurement}

Resistance was measured using standard four-point lock-in technique at $3-13 \mathrm{~Hz}$ using $0.1-1 \mathrm{nA}$ excitation current. Linear response was verified at various temperatures and magnetic fields. Measurement and filtering schematics can be found in Fig. 1d.

\section{RF filtering}

Extensive filtering minimizes electron heating caused by external radiation and improves electron thermalization. Twisted-pair signal lines are filtered at mixing chamber plate using a commercial QFilter RC/RF filter (QDevil ApS), offering over $-8 \mathrm{~dB}$ attenuation above $100 \mathrm{kHz}$ and $-50 \mathrm{~dB}$ above $300 \mathrm{MHz}$. In addition, all signal lines are filtered at room temperature by commercial in-line $\pi$-filters (API technologies corporation), providing an extra $-50 \mathrm{~dB}$ attenuation above $200 \mathrm{MHz}$. Gate voltage was applied through a roomtemperature-filtered twisted-pair well thermally-anchored at mixing chamber plate. Sample phonon temperature is measured by a calibrated on-chip ruthenium-oxide $\left(\mathrm{RuO}_{2}\right)$ thermometer positioned close to the sample.

\section{DATA AVAILABILITY}

The data that support the findings of this study are available from the corresponding author upon reasonable request.

Received: 4 November 2020; Accepted: 23 February 2021; Published online: 19 March 2021

\section{REFERENCES}

1. Kapitulnik, A., Kivelson, S. A. \& Spivak, B. Colloquium: Anomalous metals: failed superconductors. Rev. Mod. Phys. 91, 011002 (2019).

2. Breznay, N. P. \& Kapitulnik, A. Particle-hole symmetry reveals failed superconductivity in the metallic phase of two-dimensional superconducting films. Sci. Adv. 3, e1700612 (2017). 
3. Wang, Y., Tamir, I., Shahar, D. \& Armitage, N. P. Absence of cyclotron resonance in the anomalous metallic phase in $\operatorname{InO}_{x}$. Phys. Rev. Lett. 120, 167002 (2018).

4. Yazdani, A. \& Kapitulnik, A. Superconducting-insulating transition in twodimensional a-MoGe thin films. Phys. Rev. Lett. 74, 3037-3040 (1995).

5. Ephron, D., Yazdani, A., Kapitulnik, A. \& Beasley, M. R. Observation of quantum dissipation in the vortex state of a highly disordered superconducting thin film. Phys. Rev. Lett. 76, 1529-1532 (1996).

6. Mason, N. \& Kapitulnik, A. Dissipation effects on the superconductor-insulator transition in 2d superconductors. Phys. Rev. Lett. 82, 5341-5344 (1999).

7. Mason, N. \& Kapitulnik, A. True superconductivity in a two-dimensional superconducting-insulating system. Phys. Rev. B 64, 060504(R) (2001).

8. Qin, Y., Vicente, C. L. \& Yoon, J. Magnetically induced metallic phase in superconducting tantalum films. Phys. Rev. B 73, 100505(R) (2006).

9. Eley, S., Gopalakrishnan, S., Goldbart, P. M. \& Mason, N. Approaching zerotemperature metallic states in mesoscopic superconductor-normalsuperconductor arrays. Nat. Phys. 8, 59-62 (2012).

10. Saito, Y., Kasahara, Y., Ye, J., Iwasa, Y. \& Nojima, T. Metallic ground state in an iongated two-dimensional superconductor. Science 350, 409-413 (2015).

11. Bøttcher, C. G. L. et al. Superconducting, insulating and anomalous metallic regimes in a gated two-dimensional semiconductor-superconductor array. Nat. Phys. 14, 1138-1144 (2018).

12. Chen, Z. et al. Carrier density and disorder tuned superconductor-metal transition in a two-dimensional electron system. Nat. Commun. 9, 4008 (2018).

13. Couëdo, F. et al. Dissipative phases across the superconductor-to-insulator transition. Sci. Rep. 6, 35834 (2016).

14. Mason, N. \& Kapitulnik, A. Superconductor-insulator transition in a capacitively coupled dissipative environment. Phys. Rev. B 65, 220505(R) (2002).

15. Shimshoni, E., Auerbach, A. \& Kapitulnik, A. Transport through quantum melts. Phys. Rev. Lett. 80, 3352-3355 (1998).

16. Ghosal, A., Randeria, M. \& Trivedi, N. Role of spatial amplitude fluctuations in highly disordered s-wave superconductors. Phys. Rev. Lett. 81, 3940-3943 (1998).

17. Dubi, Y., Meir, Y. \& Avishai, Y. Nature of the superconductor-insulator transition in disordered superconductors. Nature 449, 876-880 (2007).

18. Aronov, A. G. \& Spivak, B. Z. Stability of nonequilibrium states of superconductors with respect to finite fluctuations. Phys. Lett. A 78, 391-394 (1980).

19. Tamir, I. et al. Sensitivity of the superconducting state in thin films. Sci. Adv. 5, eaau3826 (2019)

20. Dutta, S. et al. Extreme sensitivity of the vortex state in a-MoGe films to radiofrequency electromagnetic perturbation. Phys. Rev. B 100, 214518 (2019).

21. Feigel'man, M. V. \& Larkin, A. I. Quantum superconductor-metal transition in a $2 d$ proximity-coupled array. Chem. Phys. 235, 107-114 (1998).

22. Spivak, B., Zyuzin, A. \& Hruska, M. Quantum superconductor-metal transition. Phys. Rev. B 64, 132502 (2001).

23. Spivak, B., Oreto, P. \& Kivelson, S. A. Theory of quantum metal to superconductor transitions in highly conducting systems. Phys. Rev. B 77, 214523 (2008).

24. Steiner, M. A., Boebinger, G. \& Kapitulnik, A. Possible field-tuned superconductorinsulator transition in high- $T_{c}$ superconductors: implications for pairing at high magnetic fields. Phys. Rev. Lett. 94, 107008 (2005).

25. Beloborodov, I. S., Lopatin, A. V., Vinokur, V. M. \& Efetov, K. B. Granular electronic systems. Rev. Mod. Phys. 79, 469-518 (2007).

26. Hen, B., Zhang, X., Shelukhin, V., Kapitulnik, A. \& Palevski, A. Superconductorinsulator transition in two-dimensional indium-indium-oxide composite. Proc. Natl Acad. Sci. USA 118, e2015970118 (2021).

27. Sambandamurthy, G., Engel, L. W., Johansson, A. \& Shahar, D. Superconductivityrelated insulating behavior. Phys. Rev. Lett. 92, 107005 (2004).

28. Steiner, M. \& Kapitulnik, A. Superconductivity in the insulating phase above the field-tuned superconductor-insulator transition in disordered indium oxide films. Phys. C. Supercond. 422, 16-26 (2005).

29. Breznay, N. P., Steiner, M. A., Kivelson, S. A. \& Kapitulnik, A. Self-duality and a hallinsulator phase near the superconductor-to-insulator transition in indium-oxide films. Proc. Natl Acad. Sci. USA 113, 280-285 (2016).

30. Baturina, T. I., Strunk, C., Baklanov, M. R. \& Satta, A. Quantum metallicity on the high-field side of the superconductor-insulator transition. Phys. Rev. Lett. 98, 127003 (2007).

31. Feigel'man, M. V., Geshkenbein, V. B. \& Larkin, A. I. Pinning and creep in layered superconductors. Phys. C. Supercond. 167, 177-187 (1990).

32. Kapitulnik, A., Mason, N., Kivelson, S. A. \& Chakravarty, S. Effects of dissipation on quantum phase transitions. Phys. Rev. B 63, 125322 (2001)

33. Ando, Y., Boebinger, G. S., Passner, A., Kimura, T. \& Kishio, K. Logarithmic divergence of both in-plane and out-of-plane normal-state resistivities of superconducting $\mathrm{La}_{2-x} \mathrm{Sr}_{x} \mathrm{CuO}_{4}$ in the zero-temperature limit. Phys. Rev. Lett. 75, 4662-4665 (1995).

34. Mulligan, M. \& Raghu, S. Composite fermions and the field-tuned superconductor-insulator transition. Phys. Rev. B 93, 205116 (2016).
35. Yang, C. et al. Intermediate bosonic metallic state in the superconductor-insulator transition. Science 366, 1505-1509 (2019).

36. Wyatt, A. F. G., Dmitriev, V. M., Moore, W. S. \& Sheard, F. W. Microwave-enhanced critical supercurrents in constricted tin films. Phys. Rev. Lett. 16, 1166-1169 (1966).

37. Dayem, A. H. \& Wiegand, J. J. Behavior of thin-film superconducting bridges in a microwave field. Phys. Rev. 155, 419-428 (1967).

38. Notarys, H. A., Yu, M. L. \& Mercereau, J. E. Josephson effects at high current density. Phys. Rev. Lett. 30, 743-746 (1973).

39. Warlaumont, J. M., Brown, J. C., Foxe, T. \& Buhrman, R. A. Microwave-enhanced proximity effect in superconductor-normal-metal-superconductor microjunctions. Phys. Rev. Lett. 43, 169-172 (1979).

40. Klapwijk, T. M. \& de Visser, P. J. The discovery, disappearance and re-emergence of radiation-stimulated superconductivity. Ann. Phys. 417, 168104 (2020).

41. Eliashberg, G. Film superconductivity stimulated by a high-frequency field. JETP Lett. 11, 114-116 (1970).

42. Wilks, C. W., Bojko, R. \& Chaikin, P. M. Field dependence of the resistive transition for a square wire network. Phys. Rev. B 43, 2721-2725 (1991).

43. Steinmann, R., P, L., Chaussy, J. \& Pannetier, B. Magnetoresistance oscillations in bulk high- $T_{c}$ superconductor. Phys. C. Supercond. 153, 1487-1488 (1988).

44. Patel, $U$. et al. Magnetoresistance oscillations in superconducting granular niobium nitride nanowires. Phys. Rev. B 80, 012504 (2009).

45. Wang, J. et al. Magnetoresistance oscillations of ultrathin Pb bridges. Nano Res. 2 , 671-677 (2009).

46. Philippi, M., Gutiérrez-Lezama, I., Ubrig, N. \& Morpurgo, A. F. Lithium-ion conducting glass ceramics for electrostatic gating. Appl. Phys. Lett. 113, 033502 (2018).

\section{ACKNOWLEDGEMENTS}

We acknowledge discussions with Boris Spivak, Steven Kivelson, Sri Raghu, and Teun Klapwijk. Work at Stanford University was supported by the National Science Foundation Grant NSF-DMR-1808385. Work at Tel-Aviv University was supported by the US-Israel Binational Science Foundation (Grant No. 2014098). We thank Sejoon Lim for assistance with SEM. Part of this work was performed at the Stanford Nano Shared Facilities (SNSF), supported by the National Science Foundation under award ECCS-1542152.

\section{AUTHOR CONTRIBUTIONS}

X.Z., B.H., A.P., and A.K. designed the experiments. X.Z. grew the samples and performed measurements. X.Z. and A.K. analyzed the data and wrote the manuscript.

\section{COMPETING INTERESTS}

The authors declare no competing interests.

\section{ADDITIONAL INFORMATION}

Supplementary information The online version contains supplementary material available at https://doi.org/10.1038/s41535-021-00329-2.

Correspondence and requests for materials should be addressed to X.Z.

Reprints and permission information is available at http://www.nature.com/ reprints

Publisher's note Springer Nature remains neutral with regard to jurisdictional claims in published maps and institutional affiliations.

Open Access This article is licensed under a Creative Commons Attribution 4.0 International License, which permits use, sharing, adaptation, distribution and reproduction in any medium or format, as long as you give appropriate credit to the original author(s) and the source, provide a link to the Creative Commons license, and indicate if changes were made. The images or other third party material in this article are included in the article's Creative Commons license, unless indicated otherwise in a credit line to the material. If material is not included in the article's Creative Commons license and your intended use is not permitted by statutory regulation or exceeds the permitted use, you will need to obtain permission directly from the copyright holder. To view a copy of this license, visit http://creativecommons.org/licenses/by/4.0/.

(c) The Author(s) 2021 